\title{
ARTICLE Novel role of gastrin releasing peptide-mediated signaling in the host response to influenza infection
}

\author{
Kari Ann Shirey ${ }^{1}$, Mary E. Sunday ${ }^{2}$, Wendy Lai ${ }^{1}$, Mira C. Patel ${ }^{3}$, Jorge C. G. Blanco ${ }^{3}$, Frank Cuttitta ${ }^{4}$ and Stefanie N. Vogel ${ }^{1}$
}

\begin{abstract}
Gastrin-releasing peptide (GRP) is an evolutionarily well-conserved neuropeptide that was originally recognized for its ability to mediate gastric acid secretion in the gut. More recently, however, GRP has been implicated in pulmonary lung inflammatory diseases including bronchopulmonary dysplasia, chronic obstructive pulmonary disease, emphysema, and others. Antagonizing GRP or its receptor mitigated lethality associated with the onset of viral pneumonia in a well-characterized mouse model of influenza. In mice treated therapeutically with the small-molecule GRP inhibitor, NSC77427, increased survival was accompanied by decreased numbers of GRP-producing pulmonary neuroendocrine cells, improved lung histopathology, and suppressed cytokine gene expression. In addition, in vitro studies in macrophages indicate that GRP synergizes with the prototype TLR4 agonist, lipopolysaccharide, to induce cytokine gene expression. Thus, these findings reveal that GRP is a previously unidentified mediator of influenza-induced inflammatory disease that is a potentially novel target for therapeutic intervention.
\end{abstract}

Mucosal Immunology (2019) 12:223-231; https://doi.org/10.1038/s41385-018-0081-9

\section{INTRODUCTION}

Bombesin (BN) and gastrin-releasing peptide (GRP) are homologous amphibian/mammalian peptides, respectively, shown initially to up-regulate gastrin release and subsequent gastric acid secretion in the gut (reviewed in refs. ${ }^{1,2}$ ). Tissue distribution of BN-like (BNL) or GRP immunoreactivities have been identified in the gastrointestinal tract, pancreas, adrenals, thyroid, brain, and lung. ${ }^{2}$ The genes for GRP and its respective receptor (GRPR/BB2) have been cloned and their anatomical expression evaluated in healthy and disease states (reviewed in ref. ${ }^{1}$ ). GRP is produced as an inactive "proform" that, after cleavage and amidation of the C-terminus, becomes the "mature," active form of GRP (reviewed in ref. ${ }^{1}$ ). GRP/GRPR interaction has been demonstrated to mediate a variety of signal transduction pathways that include CAMP, MAPK, PI3K, and Akt, either directly or indirectly through the transactivation of other ligand/receptor systems (reviewed in ref. ${ }^{3}$ ). Several different types of GRP inhibitors and/or GRPR antagonists have been developed as exploratory tools for mechanistic studies including compounds that either sequester the ligand or inhibit receptor binding, for example, the neutralizing monoclonal antibody $2 \mathrm{~A} 11$ (MoAb 2A11), a small-molecule inhibitor (NSC77427), and receptor-blocking agents like the watersoluble peptide BW2258U89. ${ }^{4-7}$

Over the past two decades, many studies have sought to identify the involvement of GRP in pulmonary disease. Inflammatory disorders like bronchopulmonary dysplasia (BPD), chronic obstructive pulmonary disease (COPD), chronic bronchitis, emphysema, and fibrosis have been shown to have a GRP regulatory component (reviewed in refs. ${ }^{2,8-10}$ ). Distinct cells of the immune response are known to either produce or respond to GRP, underpinning macrophage activation and mast cell proliferation, migration, and degranulation (reviewed in refs. ${ }^{1,2,11}$ ). Chronic cigarette smoking has been demonstrated to elevate GRP levels in the lung and also in the host urine (reviewed in refs. ${ }^{1,11}$ ). For pulmonary neoplasms, GRP plays a major autocrine/ paracrine growth modulatory role in small-cell lung cancer (SCLC) and non-small-cell lung cancer (NSCLC/adenocarcinoma) (reviewed in refs. ${ }^{1,12}$ ).

Together, these findings have led to the hypothesis that GRP inhibitors or GRPR antagonists attenuate the severity of lung inflammation. In a primate model of $B P D$, it was revealed by Sunday et al. $^{8}$ that treatment with MoAb $2 A 11$, which only detects the mature (active, amidated) form of $\mathrm{GRP}^{4}{ }^{4}$ could abrogate the inflammation and arrest lung development characteristic of the disease. ${ }^{13}$ Similarly, in a radiation-induced model of pneumonitis/ fibrosis, lung injury was mitigated by therapeutic administration of the GRP inhibitor NSC77427, ${ }^{14}$ that like MoAb 2011, only binds to the mature form of GRP. ${ }^{6}$

We previously reported that mice that express a targeted mutation in the gene that encodes Toll-like receptor 4 (TLR4) or therapeutic treatment of wild-type (WT) mice with TLR4 antagonists (small-molecule inhibitors or neutralizing antibody) effectively blocked viral-induced lethality in an experimental model of influenza infection. ${ }^{15-19}$ Protection was associated with a blunting of the inflammatory response to infection, likely mediated by host-derived high-mobility group box-1 (HMGB1) activation of TLR4. ${ }^{16,17}$ TLR4 has also been shown to be involved in ozone-mediated and hyaluronanmediated airway hyperresponsiveness, while TLR4-deficient animals are refractory. ${ }^{20}$ More recently, ozone-induced airway hyperresponsiveness was shown to be inhibited by administration of MoAb $2 A 11$, suggesting a role for GRP as well. ${ }^{21}$ Interestingly, another GRPR peptide antagonist (RC-3095) was previously shown to inhibit

\footnotetext{
${ }^{1}$ Department of Microbiology and Immunology, University of Maryland School of Medicine, Baltimore, MD 21201, USA; ${ }^{2}$ Department of Pathology, Duke University Medical Center, Durham, NC 27710, USA; ${ }^{3}$ Sigmovir Biosystems Inc., Rockville, MD 20850, USA and ${ }^{4}$ Mouse Cancer Genetics Program, National Cancer Institute, NIH, Frederick, MD 21702, USA

Correspondence: Stefanie N. Vogel (svogel@som.umaryland.edu)
}

Received: 16 March 2018 Revised: 19 July 2018 Accepted: 8 August 2018

Published online: 16 October 2018 
TLR4 signaling and proposed as a possible therapeutic intervention strategy in sepsis. ${ }^{22}$ Conversely, the low-affinity GRPR (NMBR/BB1) was shown to mediate macrophage Neu1 sialidase and matrix metalloproteinase-9 (MMP9) cross-talk inducing the transactivation of TLR-like receptors and cellular signaling. ${ }^{23}$

Based on these collective findings, we evaluated two GRP inhibitors and one GRPR antagonist for their effectiveness in suppressing host lethality associated with the onset of viral pneumonia in a well-characterized mouse model of influenza. Influenza-induced lethality was blunted significantly by both the GRP inhibitors and the GRPR antagonist, and survival was accompanied by decreased numbers of GRP-producing pulmonary neuroendocrine cells (PNECs), improved lung histopathology, and suppressed proinflammatory cytokine gene expression. In addition, in vitro studies in macrophages indicate that GRP synergizes with the prototype TLR4 agonist, lipopolysaccharide (LPS), to induce cytokine gene expression. Together, these findings support the hypothesis that GRP contributes to influenza-induced disease.

\section{RESULTS}

Influenza infection induces GRP production in mice

WT C57BL/6J mice were infected intranasally (i.n.) with an $\sim \mathrm{LD}_{90}$ of a mouse-adapted influenza strain, A/PR/8/34 (PR8). ${ }^{16}$ Mice were euthanized on days $2,4,6$, and 8 post infection and the lungs harvested and homogenized for measurement of GRP levels by enzyme immunoassay (EIA). The level of GRP rose significantly in the lungs of PR8-infected mice starting 4 days post-infection and increased through day 8 post infection (Fig. 1a). Cotton rats (Sigmodon hispidus) have been used as an experimental model for human respiratory virus infections and are uniquely susceptible to non-adapted human strains of influenza. ${ }^{24}$ GRP levels were measured in the sera of cotton rats in response to three influenza A strains, California pH1N1, Wuhan H3N2, and Victoria H3N2, at different times post-infection. All three strains induced significant increases in the levels of serum GRP over time before returning to basal levels by day 14 (Fig. 1b).

Therapeutic administration of GRP inhibitors protect against PR8induced lethality in mice

To determine if antagonism of GRP or the GRPR during PR8 infection would affect survival of mice, WT C57BL/6J mice were infected with PR8 $\left(\sim \mathrm{LD}_{90}\right)$ on day 0 . On day 2 post-infection, the GRP small-molecule inhibitor, NSC77427, was administered once daily for 5 consecutive days. Mice were monitored daily for survival. Mice treated with the NSC77427 were significantly protected from PR8-induced lethality $(53.3 \%$ survival, while only $\sim 7 \%$ of mice treated with vehicle survived after infection $(p<$ 0.0002 ; Fig. 2a). Similar results were seen in mice infected with a non-adapted human strain, A/California/07/2009 H1N1, and treated with NSC77427 (Supplemental Fig. 1). To confirm these findings, mice were treated with a highly specific anti-GRP

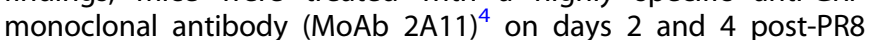
infection. Anti-GRP $\operatorname{lgG} 1 \mathrm{~K}(100 \mu \mathrm{g} /$ mouse), but not an isotype control lgG1к (MOPC21), protected mice against lethal infection $(50 \%$ survival; $p<0.0001)$, confirming the protection observed with NSC77427 (Fig. 2b). In addition, the efficacy of treatment of influenza-infected mice with the small-molecule GRPR antagonist, BW2258U89, was similarly evaluated. Therapeutic administration of the GRPR antagonist once daily from days 2 to 6 post infection resulted in $\sim 60 \%$ survival compared to treatment with vehicle alone (10\%) $(p<0.033$; Fig. 2c). Taken together, these data strongly support a role for GRP in the lethal response to influenza infection.

GRP antagonism ameliorates the lung inflammatory response to influenza infection

Therapeutic treatment of PR8-infected mice with the smallmolecule GRP inhibitor, NSC77427, also led to a reduction in influenza-induced lung pathology (Fig. 3), in the absence of a significant change in $\log _{10}$ viral titers $(6.84 \pm 0.1$ tissue culture infectious dose $50 \%\left(\mathrm{TCID}_{50}\right)$ in PR8-infected, vehicle-treated mice vs. 6.01 $\pm 0.4 \mathrm{TCID}_{50}$ in PR8-infected, NSC77427-treated mice). Briefly, groups of mice were infected with PR8 and subsequently treated with vehicle or NSC77427 from days 2 to 6 post-infection as described for Fig. 2a. Mice were euthanized on day $6,3 \mathrm{~h}$ after the final treatment. Mock-infected, vehicle-treated mice had normal lung architecture with intact airway epithelium, clear bronchi/bronchioles, and alveoli (Fig. 3a). Mice infected with influenza PR8 developed two distinct populations of inflammatory infiltrates: moderate collections of mononuclear cells surrounding conducting airways, mainly large-sized and medium-sized bronchioles, and dense nests of neutrophils in colony-like structures throughout the alveoli (Fig. 3b). At higher magnification (Fig. 3c), neutrophils are seen invading the alveoli in a vasculo-centric distribution, often in continuity with the pleural surface. PR8infected mice that were treated with NSC77427 had either no inflammation, minimal collections of mononuclear cells surrounding conducting airways, or scattered neutrophils without the formation of nests and without evidence of alveolar destruction (Fig. 3d). The mean lung injury score based on blinded histopathology, as described in the Materials and methods section, was significantly different between mice infected and treated with vehicle only vs. mice infected then treated with NSC77427 (Fig. 3e; $p=0.002$ ).

In the lung, PNECs are the classical cell type that produces GRP. ${ }^{25}$ GRP is not visualized in normal PNECs in the mouse in the absence of oxidative stress or inflammation. ${ }^{25}$ To assess whether PNEC numbers increased following PR8 infection, we used immunohistochemical (IHC) staining techniques that targeted two select neural/neuroendocrine marker antigens consisting of
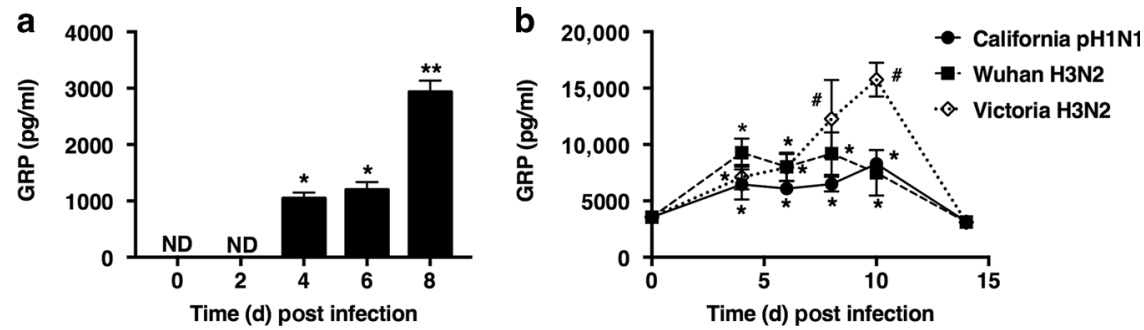

Fig. 1 Influenza infection of mice and cotton rats induces GRP in lungs and serum. a WT C57BL/6J mice were infected with mouse-adapted influenza strain PR8 (LD $\left.90 ; \sim 7500 \mathrm{TCID}_{50}\right)$. Mice were euthanized on days $0,2,4,6$, and 8 post-infection $\left(3-5\right.$ mice/group; ${ }^{*} p<0.001$; ${ }^{* *} p<0.0002$ ). Lungs were homogenized and processed for GRP levels by EIA according to the manufacturer's protocol. $\mathbf{b}$ Cotton rats were infected i.n. with $1 \times 10^{6} \mathrm{TCID}_{50}$ of either California pH1N1, and Wuhan H3N2, or $1 \times 10^{5} \mathrm{TCID}_{50}$ of Victoria H3N2, and serum GRP levels were analyzed at the indicated days p.i. ${ }^{*} p<0.05$ and $p<0.01$ for comparison between day $0 \mathrm{GRP}(N=23)$ vs. each time p.i. for each of the different influenza A strains 

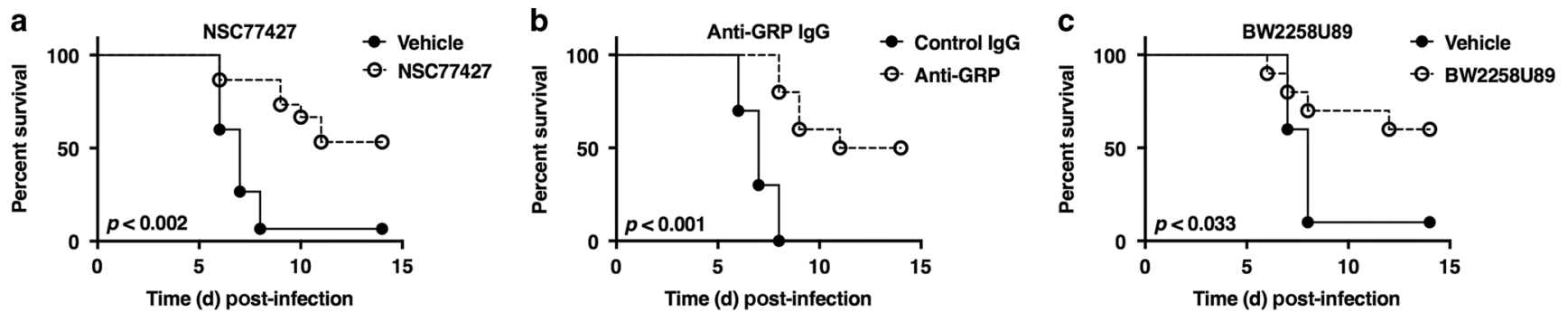

Fig. 2 Blocking GRP and GRPR enhances survival after influenza infection. a WT C57BL/6J mice were infected with mouse-adapted influenza strain PR8 ( $\mathrm{LD}_{90} ;$ 7500 TCID 50 ). Mice received vehicle (saline + 0.096\% DMSO) or GRP inhibitor (NSC77427; $20 \mu \mathrm{M}, 100 \mu \mathrm{l}$ i.v./mouse) daily from day 2 to day 6 post-infection. Survival was monitored for 14 days. Data shown are combined results of three assays (5 mice/treatment group/ experiment). b WT C57BL/6J mice were infected as described in a. Mice received either control lgG or a highly specific anti-GRP IgG (100 $\mu$; $100 \mu \mathrm{l}$ i.v./mouse) on days 2 and day 4 post-PR8 infection. Survival was monitored as in a. Data shown are combined results of two assays (5 mice/treatment group/experiment). c WT C57BL/6J mice were infected as described in a. Mice received vehicle (saline) or GRPR antagonist (BW2258U89; $20 \mu \mathrm{M}, 100 \mu \mathrm{l}$ i.v./mouse). Survival was monitored as in a. Data shown are combined results of two assays (5 mice/treatment group/experiment)
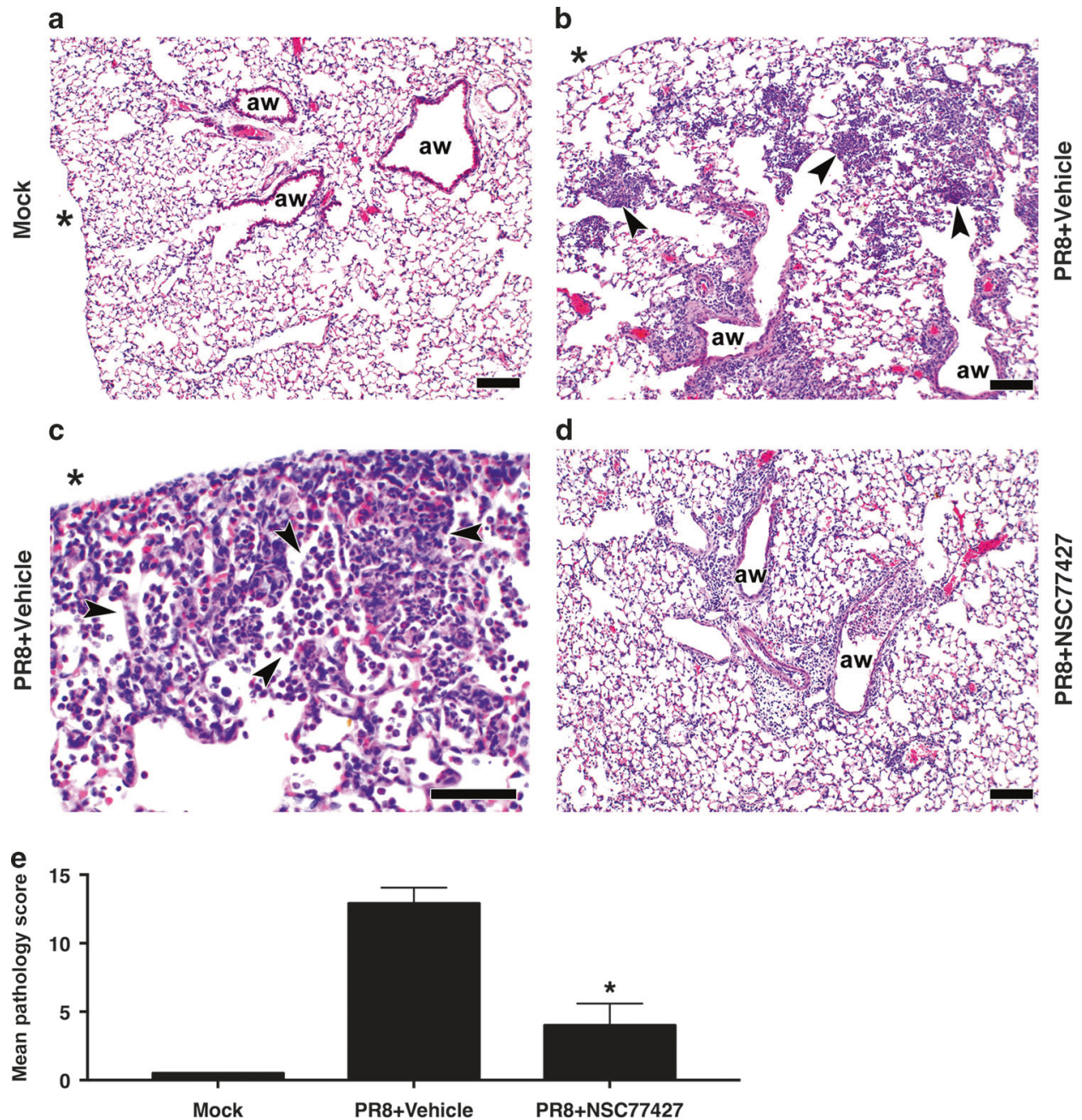

Fig. 3 GRP inhibitor, NSC77427, reduces lung pathology after influenza PR8 challenge. WT C57BL/6J mice (5 mice/treatment group) were infected i.n. with mouse-adapted influenza strain PR8 (LD $90 ; \sim 7500$ TCID $_{50}$ ). Mice received vehicle (saline $+0.0096 \%$ DMSO) or GRP inhibitor (NSC77427; $20 \mu \mathrm{M}$ /mouse; i.v.) daily from days 2 to 6 post-infection. On day 7 post-infection, mice were euthanized and lungs were extracted, fixed, and stained for histopathology. Photomicrographs of representative sections were taken at $\times 10$ (a, b-d), and at $\times 40$ (c). All scale bars are $100 \mu \mathrm{m} . N=4 \mathrm{mice} / \mathrm{group}$. Asterisk indicates the pleural surface. e Quantitation of lung injury is based on the scoring system detailed in the Materials and methods section. Data shown are mean \pm SD. ${ }^{*} p=0.002$ 
protein gene product 9.5 (PGP 9.5, cytoplasmic marker for ubiquitin hydrolase) and fully mature amidated GRP (Fig. 4). In naive or vehicle-injected, uninfected mice, infrequent PGP 9.5+ PNECs, GRP - cells are observed in conducting airways, usually bronchioles (Fig. 4a), consistent with observations in other laboratories. ${ }^{25}$ PGP $9.5+$ intrapulmonary nerves are also visible (asterisk in Fig. 4a), but all pulmonary nerves are GRP - (Fig. 4b). At 6 days post-PR8 infection (Fig. 4c), there is $~ 6-8$-fold increase in the number of stainable PGP+ PNECs (image analysis in Fig. 4g), together with an $\sim 6$-fold increase in the appearance of GRP+
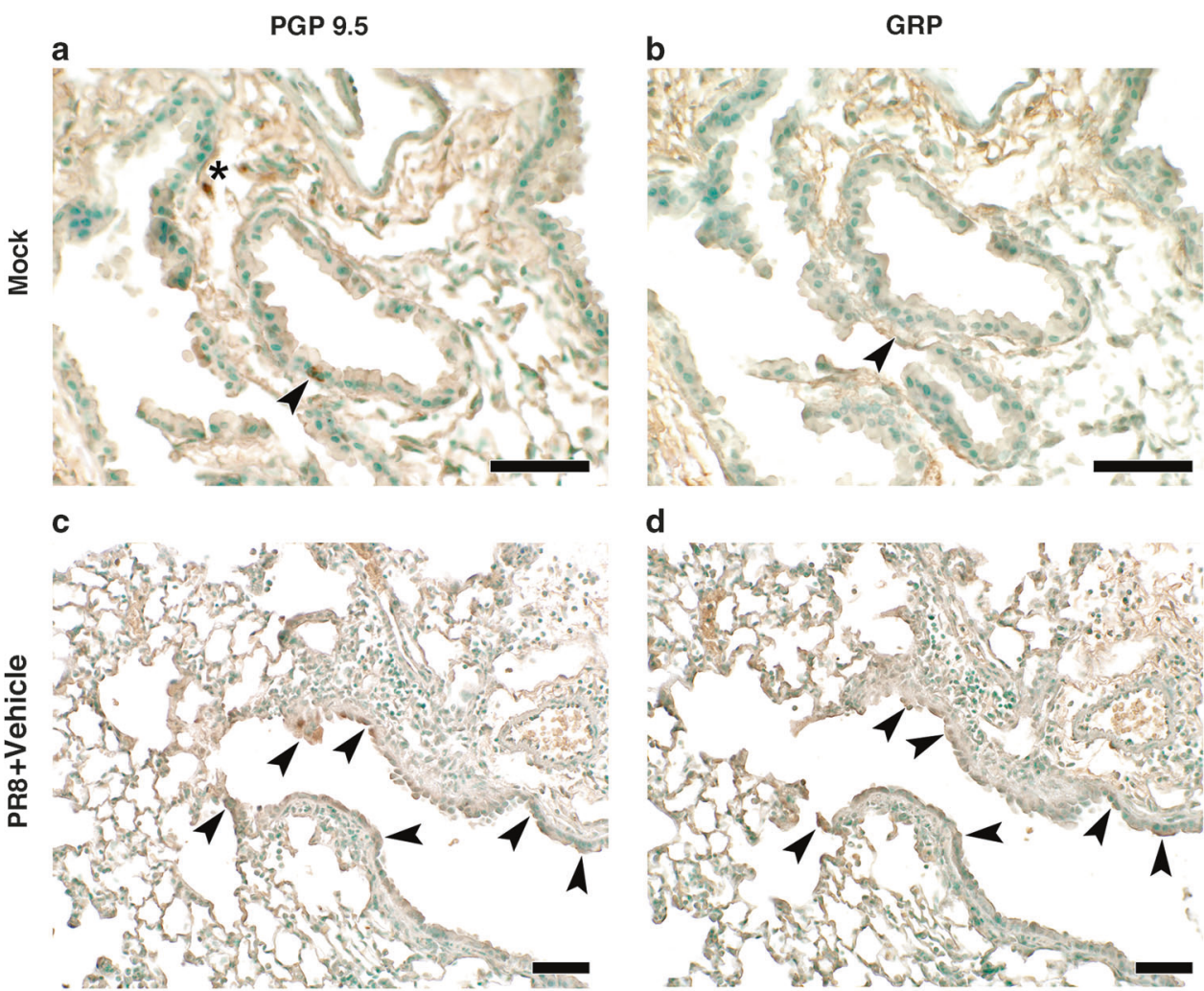

d
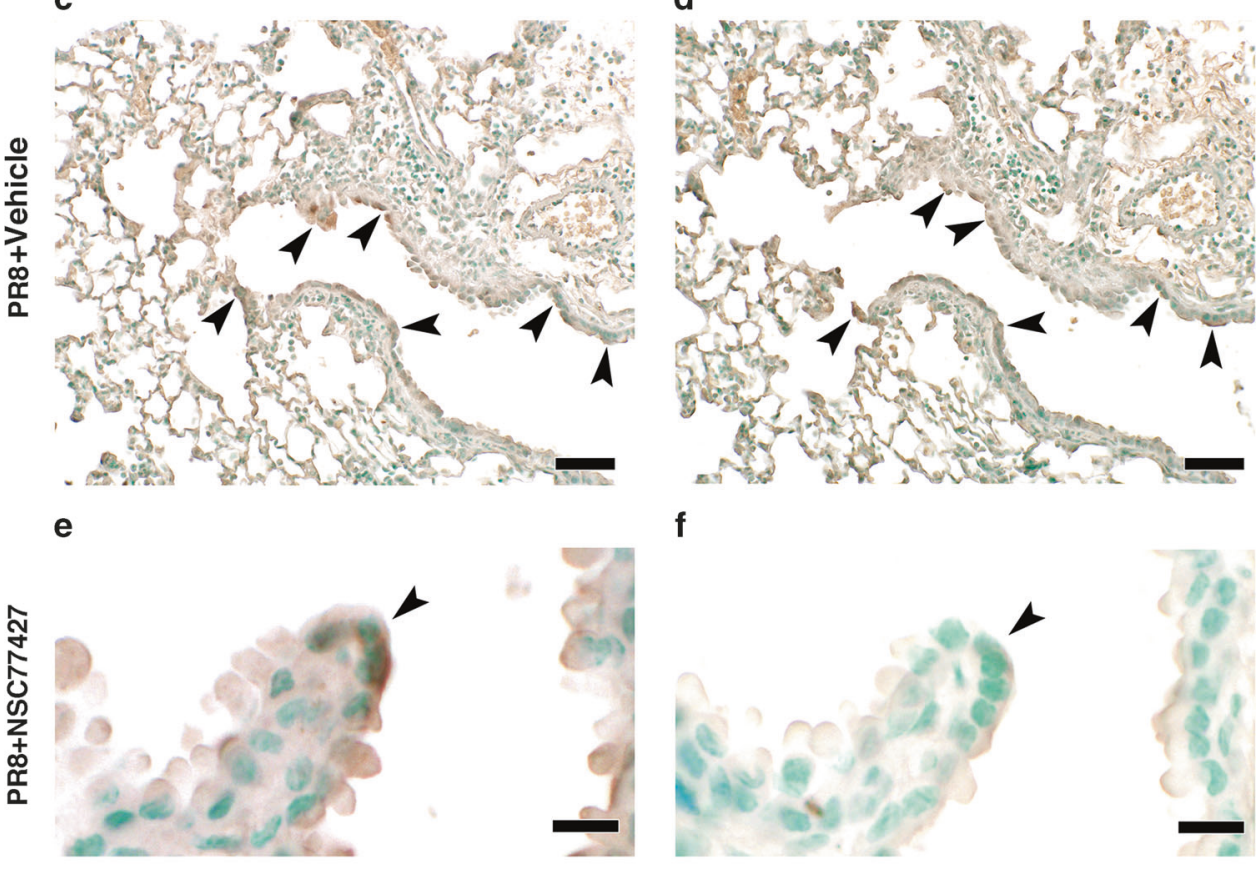

f

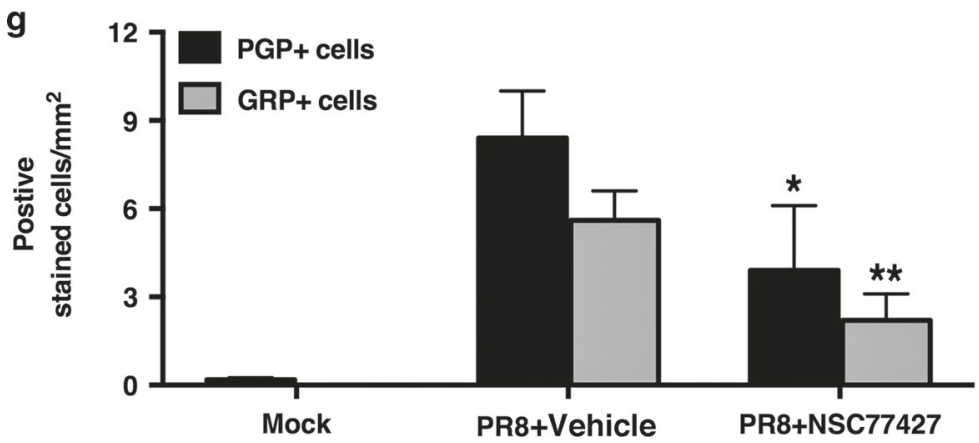

Fig. 4 IHC staining for pulmonary neuroendocrine cells (PNECs), PGP 9.5, and GRP in influenza-infected mice. Lung sections from Fig. 3 were stained in a mock-infected, c PR8 + vehicle, and e PR8 + NSC77427 for neural/neuroendocrine-specific PGP 9.5, or in b mock-infected, d PR8 + vehicle, and $\mathbf{f}$ PR8 + NSC77427 for GRP, a neuroendocrine granule marker. Scale bars shown are $50 \mu \mathrm{m}$ for $\mathbf{a}-\mathbf{d}$, and $10 \mu \mathrm{m}$ for $\mathbf{e}$ and $\mathbf{f}$. Arrows are positioned in each panel within a treatment group to indicate groups of positive cells, except in $\mathbf{b}$, where the arrow indicates GRP- cells adjacent to the PGP 9.5+ cells shown in a. g Graphic image analysis of PGP 9.5+ and GRP+ cells after IHC staining. Slides were blinded by an observer with no knowledge of experimental groups. PCP9.5+ and GRP+ cells were counted throughout each complete lung lobe crosssection for each animal, with validation by $\times 40$ micrographs of each slide. Total tissue area was determined by using Imagel thresholding analysis (methyl green counterstain) because PNECs are mainly observed in alveolar ducts and small bronchioles. ${ }^{*} p=0.009 ;{ }^{* *} p=0.001$ 
PNECs (Fig. 4d; image analysis in Fig. 4g), both primarily in the form of linear hyperplasia in small bronchioles. In PR8-infected mice, there is also extracellular GRP immunostaining consistent with GRP secretion. Mice infected with PR8, then treated with NSC77427 (Fig. 4e, f), had $~ 50 \%$ fewer PGP+ PNECs $(p=0.009)$, an $\sim 70 \%$ decrease in GRP+ PNECs $(p=0.001)$ (image analysis in Fig. $4 \mathrm{~g}$ ) and visibly less GRP immunostaining in the extracellular matrix (Fig. 4f). These observations suggest that GRP may contribute to PNEC differentiation and/or its own synthesis, consistent with observations in fetal baboon lung (reviewed in ref. ${ }^{11}$ ). Taken together, these observations indicate that antagonism of GRP function during PR8 infection results in a decrease in both the total number of PNECs and GRP production by the cells.

Effect of GRP antagonism on TLR4 signaling in macrophages We have previously shown that PR8 infection of mice elicits a strong inflammatory response characterized by a significant upregulation of cytokine and chemokine gene expression. ${ }^{16}$ The lungs of PR8-infected mice treated with the NSC77427 also showed blunted mRNA expression for genes that encode the proinflammatory cytokines interleukin-1 $\beta$ (IL-1 $\beta$ ) and tumor necrosis factor- $\alpha$ (TNF- $\alpha$ ), as well as interferon regulatory factor-3 (IRF-3)-dependent genes that encode interferon- $\beta$ (IFN- $\beta$ ), and the chemokine, regulated upon activation normal $T$ cell expressed and secreted (RANTES) (Fig. 5). Similar results were observed at the level of cytokine protein levels in lung homogenates from PR8infected, NSC77427-treated mice (Supplemental Fig. 2).

Petronilho et al. ${ }^{22}$ previously showed that a different GRPR inhibitor than used in our study, RC-3095, reduced TLR4 mRNA expression, blunted TLR4 signaling, and reduced production of IL6 and monocyte chemoattractant protein-1 (MCP-1) in the RAW 264.7 murine macrophage cell line stimulated with the prototype TLR4 agonist, LPS. In addition, they found that RC-3095, when administered immediately after surgery, inhibited cytokine/chemokine production in rats in a model of polymicrobial sepsis induced by cecal ligation and puncture (CLP). These authors correlated serum GRP levels with disease severity in septic patients. Since we previously reported that $\mathrm{TLR}^{-1-}$ mice are refractory to PR8 infection, ${ }^{15,16}$ and have shown that multiple TLR4 antagonists protect WT mice from lethal PR8 infection, ${ }^{16-19}$ we initially sought to determine if the GRP inhibitor, NSC77427, would exert an inhibitory effect on TLR4 signaling. WT mouse primary macrophages were pretreated with the GRP inhibitor, NSC77427, for $1 \mathrm{~h}(0.5 \mu \mathrm{M}$, a dose of NSC77427 that blocked GRP-induced angiogenesis in vitro and in vivo), ${ }^{7}$ followed by LPS stimulation for $2 \mathrm{~h}$. NSC77427 did not block LPS-induced MyD88-dependent (IL$1 \beta$, TNF- $\alpha$ ) or TRIF-dependent (IFN- $\beta$, RANTES) gene expression (Supplemental Fig. 3A). Likewise, treatment of macrophages with the GRPR antagonist, BW2258U89 $(1 \mu \mathrm{M})$, for $1 \mathrm{~h}$ prior to LPS stimulation had no effect on LPS signaling (Supplemental Fig. 3B). These data suggest either that the effect of GRP is not directly on macrophages or that macrophages are not the primary source of GRP, as supported by our IHC staining data (Fig. 4g). Since macrophages have been shown to express the $\mathrm{GRPR}^{26}$ we next sought to test the hypothesis that exogenous GRP would modulate TLR4-dependent cytokine gene expression. To this end, WT macrophages were treated with low doses of LPS (0.1 and $1 \mathrm{ng} / \mathrm{ml}$, respectively) in the absence or presence of increasing doses of GRP (1-100 $\mathrm{nM}$ ) for $2 \mathrm{~h}$ and gene expression measured. While these concentrations of GRP alone again did not induce cytokine gene expression, addition of GRP to suboptimal doses of LPS resulted in synergistic induction of both MyD88-dependent (IL-1 $\beta$ and TNF- $\alpha$ ) and TRIF-dependent (MyD88-independent; IFN- $\beta$ and RANTES) cytokine gene expression (Fig. 6). This synergistic effect was largely lost at higher doses of LPS (Supplemental Fig. 4).

\section{DISCUSSION}

Over 40 years ago, the lung was characterized as a new endocrine organ that produced classic peptide hormones in distinct cells of the bronchiole columnar epithelium denoted as PNEC. ${ }^{27}$ Subsequent to this discovery, immunoreactive amphibian BNL activity was identified in human fetal PNEC and later determined to be mammalian homolog, GRP (reviewed in refs. ${ }^{11,28}$ ). Like most peptide hormones, GRP is initially produced in the cell as its inactive pre-proform, enzymatically processed in the Golgi cisterna and secretory granules, and then released to the external milieu as mature bioactive methionine-amide GRP. ${ }^{29}$ The potential for endocrine regulation of the immune response was first implicated in 1985 and has since been shown to be a dynamic
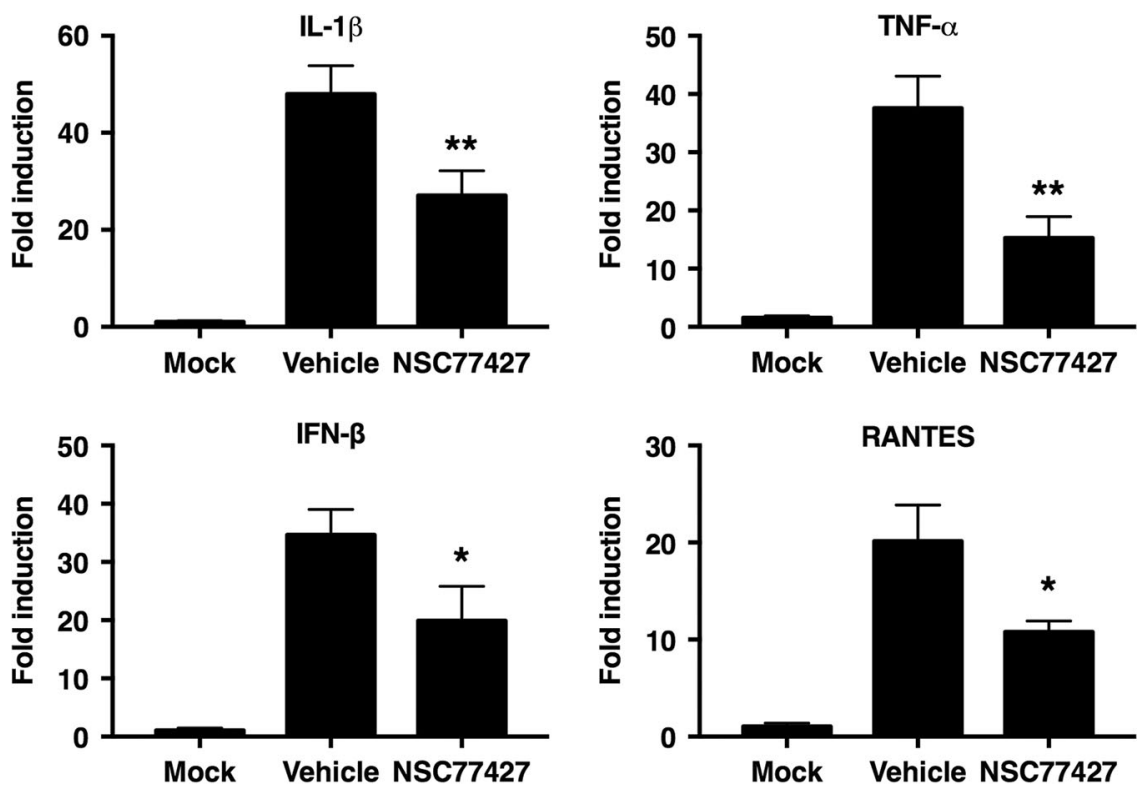

Fig. 5 Blocking GRP blunts influenza-induced cytokine induction in vivo. WT C57BL/6J mice (5 mice/treatment group) were infected i.n. with mouse-adapted influenza strain PR8 $\left(\mathrm{LD}_{90} ; \sim 7500 \mathrm{TCID}_{50}\right)$. Mice received vehicle (saline $+0.0096 \%$ DMSO) or GRP inhibitor (NSC77427; $20 \mu \mathrm{M} /$ mouse; i.v.) daily from day 2 to day 6 post infection. On day 7 post-infection, lungs were harvested and total RNA was extracted to measure gene expression by qRT-PCR. ${ }^{* *} p<0.01 ;{ }^{*} p<0.05$ 

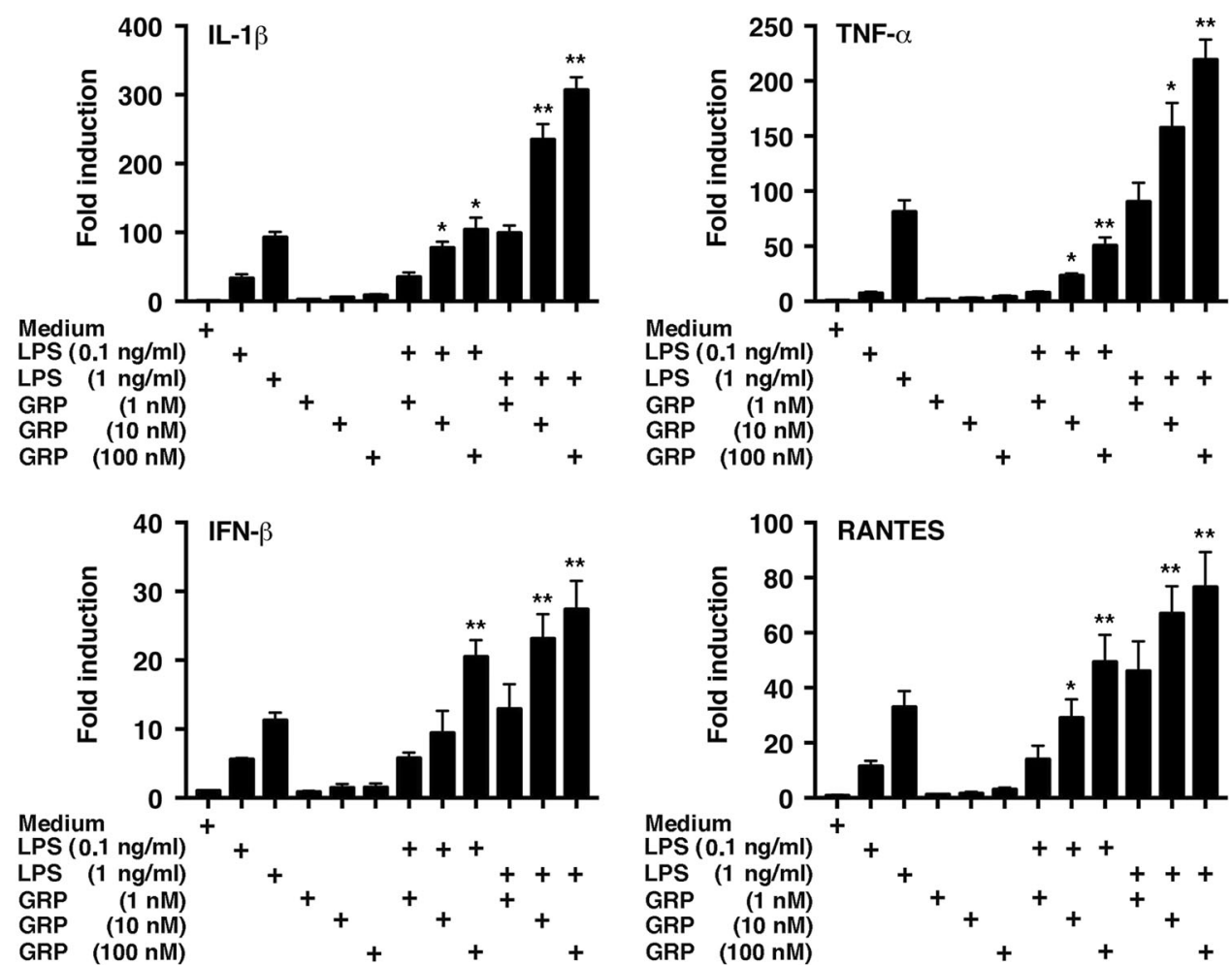

Fig. 6 GRP enhances LPS signaling. WT C57BL/6J peritoneal macrophages were treated with medium only or LPS (0.1 or $1 \mathrm{ng} / \mathrm{ml})$ in the absence or presence of increasing GRP doses $(1,10$, or $100 \mathrm{nM}$, respectively) for $2 \mathrm{~h}$ and mRNA expression measured. Data represent the means \pm SEM from two separate experiments $\left({ }^{*} p<0.01 ;{ }^{* *} p<0.001\right.$ compared to induction of gene expression with LPS treatment alone)

bidirectional process where immune cells can make and respond to peptide hormones. ${ }^{30-32}$ BNL/GRP has been shown to not only stimulate fetal lung growth/maturation, but also to contribute to a variety of pulmonary inflammatory diseases and malignancies including BPD, COPD, emphysema, fibrosis, SCLC, and NSCLCs (reviewed in refs. ${ }^{1,11,12}$ ). However, relatively little attention has been given to the potential role of GRP in lung inflammation of infectious etiology.

Influenza infection poses a global threat. Despite the availability of annual vaccines, the need to predict the major strains to be included in each upcoming year's vaccine, as well as the appearance of strains to which we have no prior exposure, has led to an ongoing effort by many to develop a "universal influenza vaccine" (reviewed in refs. ${ }^{33,34}$ ). In addition, influenza viruses have mutated to become resistant to many of the drugs in our current arsenal of antiviral agents. ${ }^{35,36}$ In addition, antiviral therapies must be administered relatively early in infection to be effective. ${ }^{37}$ Therefore, we have focused on alternative therapeutic strategies that act by modulating the host's innate immune response to influenza infection. To date, we have shown that antagonizing TLR4 signaling therapeutically is efficacious in both murine and cotton rat models of influenza. The TLR4 agonist activity induced by influenza infection is attributable, in large part, to the action of a host-derived "danger-associated molecular pattern," HMGB1. HMGB1, a chromatin-associated molecule, is often released from dying cells and has been shown to be a TLR4 agonist. ${ }^{38}$ In our current study, we demonstrate for the first time that GRP plays an active role in regulating host lethality and lung pathology in a mouse model of influenza.

The cellular mechanism underlying GRP induction during influenza infection is revealed by the IHC composites shown in Fig. 4. Our data strongly support the observation that both the number of PGP 9.5+ PNECs and the number of GRP + PNECs was increased upon infection, indicating a direct effect on the differentiation of these specialized epithelial airway cells, which has been reported to result from the interplay of increased canonical Wnt signaling and diminished Notch signaling. ${ }^{39,40}$ Together, these observations suggest that GRP may provide a "feed-forward" signal that promotes PNEC differentiation and/or its own synthesis, consistent with observations in fetal baboon lung. ${ }^{41}$ In addition, influenza infection has been shown to activate Wnt signaling, perhaps facilitating the production of GRP. ${ }^{42}$ In this regard, Shapira et al. ${ }^{42}$ found that treatment of human bronchial epithelial cells with recombinant WNT protein increased cellular production of IFN- $\beta$ in response to influenza infection.

Oxidative stress or acute lung injury can mediate PNEC hyperplasia accompanied by enhanced GRP expression in these neuroendocrine cells followed by release of this hormone into the surrounding airway parenchyma (reviewed in refs. ${ }^{11,43}$ ). GRPR, in turn, is known to mediate neutrophil chemotaxis and lung macrophages/PMN infiltrates that can produce the regional release of MMP2/MMP9. These contribute to the breakdown of basement membrane integrity and the altering of bronchiole/ alveolar architecture, diminution of pulmonary function, and the onset of disease lethality in COPD and lung fibrosis. ${ }^{26,44-46} \mathrm{We}$ hypothesize that similar pathological events occur in our animal model of influenza and targeting GRP with appropriate inhibitors/ antagonists disrupts, in part, disease progression.

Eritoran, a non-signaling molecule based on the structure of the lipid A region of LPS, ${ }^{47}$ is a potent TLR4 antagonist that blocks signaling by competitively binding to the TLR4 co-receptor, MD$2{ }^{48}$ We previously reported that therapeutic administration of Eritoran, ${ }^{16,17}$ or a structurally related TLR4 antagonist, FP7, ${ }^{19}$ to PR8-infected mice, blunted the cytokine storm and the lung pathology that is normally induced by infection. Similarly, therapeutic administration of the GRP inhibitor, NSC77427, also inhibited cytokine production in whole lung homogenates from PR8-infected mice (Fig. 5). Thus, the inflammatory response to PR8 infection appears to be mediated, in part, by the action of GRP on both PNEC differentiation and cytokine gene expression. 
To better understand the role of GRP in TLR4-mediated cytokine production, we analyzed the potential contribution of GRP to the induction of cytokine gene expression by primary peritoneal macrophages stimulated by the prototype TLR4 agonist, LPS. Petronilho et al. ${ }^{22}$ showed previously that a different GRPR antagonist than that used in our study, RC-3095, blocked LPSinduced activation of extracellular signal-related kinase-1/2, Jun $\mathrm{NH}_{2}$-terminal kinase, and Akt, as well as decreased activation of activator protein-1, nuclear factor-kB (NF-KB), and IL-6 and MCP-1 secretion induced by LPS in the RAW 264.7 murine macrophage cell line. However, the effects of RC-3095 were not restricted to TLR4 signaling since RC-3095 also blunted the IL- 6 response to TNF-a. They also reported that treatment of mice with RC-3095 immediately after CLP, a model of polymicrobial sepsis, decreased levels of MCP- 1 and IL- 6 in the serum and in the brochoalveolar lavage fluid. Finally, this study correlated elevated plasma GRP levels in sepsis patients with a worse outcome. In contrast to the Petronilho et al. study, 22 we found that the GRP inhibitor, NSC77427, failed to block LPS-induced expression of both MyD88-dependent and IRF-3-dependent genes in macrophage cultures (Supplemental Fig. 3). Conversely, high concentrations of exogenous GRP failed to induce macrophage cytokine gene expression, yet particularly at low concentrations of LPS, synergistic induction between GRP and LPS was observed (Fig. 6; Supplemental Fig. 4). These findings are more in line with the work of Meloni et al. ${ }^{49}$ who reported that BN augments procoagulant activity under conditions of suboptimal LPS-induced activation of alveolar macrophages. At this time, the mechanism by which GRP and LPS synergize is unknown; however, it is possible that TLR4 interacts with GRPR since TLR4 has been found to interact physically with another $G$ protein-coupled receptor, proteaseactivated receptor $2\left(\mathrm{PAR}_{2}\right)$, and enhances NF-KB activation in responsive to $\mathrm{PAR}_{2}$ agonist peptide. ${ }^{50}$ Abdulkhalek et al. $^{23}$ have also provided evidence that ligand activation of GRPR transactivates TLR4 through the action of Neu1 sialidase and MMP9. Additionally, GRP has been shown to transactivate EGFR under ligand-free conditions in the malignant setting. ${ }^{51-53}$ Interestingly, a number of different studies have pointed to a possible role for EGFR in TLR4-mediated signaling. ${ }^{54-59}$ Thus, the possible role of GRPR-mediated EGFR transactivation in influenza lethality remains to be determined.

Taken together, our data support the possibility that during PR8 infection other cell types, for example, PNECs, are likely to be the major source of GRP (consistent with the results of Fig. 4), and that administration of the GRP inhibitor blocks its ability to synergize with TLR4 signaling to elicit a more potent cytokine storm, leading to lung pathology and death. In conclusion, our findings suggest that GRP is a neuroendocrine peptide that acts as a novel contributor to the inflammatory response to influenza infection. Thus, inhibition of GRP or antagonizing the GRPR during influenza infection represents a novel therapeutic approach to mitigating lung damage.

\section{MATERIALS AND METHODS}

Reagents

GRP EIA, which is specific for the mature form of GRP (amide 1-27), was purchased from Phoenix Pharmaceuticals Inc. (Burlingame, CA, USA; catalog \# EK-027-07). The GRP inhibitor, NSC77427, was made by the Small Molecule Library Reagent Program (National Cancer Institute, Division of Cancer Treatment and Diagnosis/Developmental Therapeutics Program, NIH). The GRPR antagonist, BW2258U89, was purchased from Phoenix Pharmaceuticals Inc. (Burlingame, CA, USA). The anti-GRP antibody mouse monoclonal antibody, MoAb $2 A 11(\lg G 1 \mathrm{~K})$, was made by the National Cancer Institute, Center for Cancer Research, NCl-Navy Medical Branch and is also specific for the mature form of GRP. ${ }^{6}$ Mouse $\operatorname{lgG} 1 \mathrm{~K}$ isotype control antibody (MOPC21) was purchased from BioLegend (San Diego, CA, USA). Both the MOPC21 and control antibody preparations were confirmed to be endotoxinfree by a chromogenic Limulus Amoebocyte Assay (East Falmouth, MA, USA). The murine monoclonal mAb-2A11 (2A11) was provided by Dr. Cuttitta $(\mathrm{NCl}, \mathrm{NIH})$. Rabbit polyclonal anti-PGP 9.5 was obtained from Gene Technology Co., Ltd. (Shanghai, China).

Mice and cotton rats

Six- to 8-week-old, WT C57BL/6J mice were purchased from the Jackson Laboratory (Bar Harbor, ME, USA). Four- to six-week-old, male or female cotton rats were obtained from the inbred colony maintained at Sigmovir Biosystems Inc. (SBI, Rockville, MD, USA). All animal experiments were conducted with institutional IACUC approvals from the University of Maryland, Baltimore and SBI.

Viruses

Mouse-adapted H1N1 influenza A/PR/8/34 virus ("PR8") (ATCC, Manassas, VA, USA) was grown in the allantoic fluid of 10-day-old embryonated chicken eggs as described ${ }^{60}$ and was kindly provided by Dr. Donna Farber (Columbia University). Nonadapted human influenza A/California/07/2009 strain (human pandemic H1N1) was kindly provided by Ted Ross (University of Pittsburgh). Preparation of human A/California 04/2009 (stock titer: $4.3 \times 10^{7} \mathrm{TCID}_{50} / \mathrm{ml}$ ) and A/Wuhan/359/95 (stock titer: $1 \times 10^{7}$ $\mathrm{TCID}_{50} / \mathrm{ml}$ ) were previously described. ${ }^{24,61}$ Human A/Victoria H3N2 (stock titer: $6.8 \times 10^{6} \mathrm{TCID}_{50} / \mathrm{ml}$ ) virus was obtained by harvesting the supernatants of Madin-Darby canine kidney (MDCK) cells inoculated 3 days previously at a low multiplicity of infection. Titers of all virus stocks were determined by standard endpoint dilution assays on MDCK cells as previously described. ${ }^{62}$

Virus challenge and treatment

For survival experiments, WT C57BL/6J mice were infected with mouse-adapted influenza virus, strain A/PR/8/34 (PR8; 7500 $\mathrm{TCID}_{50}$, i.n., $\left.25 \mu \mathrm{l} / \mathrm{nares}\right)$, a dose of PR8 that kills $\sim 90 \%$ of infected mice. ${ }^{16,17}$ Two days after PR8 infection, mice received either vehicle (indicated in the figure legend), NSC77427 $(20 \mu \mathrm{M} ; 100 \mu \mathrm{l}$ intravenously (i.v.)), or BW2258U89 $(20 \mu \mathrm{M} ; 100 \mu$ i.v.) daily for five consecutive days (day 2 until day 6). For neutralizing antibody studies, mice received either $\lg \mathrm{G} 1 \mathrm{~K}$ isotype control antibody (100 $\mu g ; 100 \mu$ i.v.) or anti-GRP mouse MoAb 2A11 (IgG1k; $100 \mu g ;$ i.v.) on days 2 and 4 post infection. Mice were monitored daily for survival. In a separate assay, mice were infected with a nonadapted influenza strain, A/California/07/2009 H1N1 ( 10 ${ }^{7}$ TCID $_{50}$, i.n., $25 \mu \mathrm{l} /$ nares), a dose shown to kill $\sim 75 \%$ of infected mice. ${ }^{16}$ Two days after PR8 infection, mice received either vehicle or NSC77427 $(20 \mu \mathrm{M} ; 100 \mu \mathrm{l}$ i.v.) daily for 5 consecutive days (day 2 until day 6). Mice were monitored daily for survival for 14 days. In some experiments, mice infected with PR8 were euthanized at days 2, 4, 6 , or 8 post infection to harvest lungs for analysis of GRP protein levels by EIA, or for gene expression lung pathology, and IHC.

For cotton rat experiments, groups of five animals were infected as follows: California pH1N1 and Wuhan H3N2 $\left(1 \times 10^{6} \mathrm{TCID}_{50}\right)$, and with Victoria H3N2 $\left(1 \times 10^{5} \mathrm{TCID}_{50}\right)$. Serum GRP levels were analyzed at $0,4,6,8,10$, and 14 days post infection using the same GRP EIA Kit used for mouse studies.

\section{Histology and staining}

Lungs were inflated and perfused and fixed with 4\% paraformaldehyde. Fixed sections $(5 \mu \mathrm{m})$ of paraffin-embedded lungs were stained with hematoxylin and eosin.

Scoring of blinded slides by a board-certified pathologist was performed for severity of lung injury utilized multiple parameters, which were then added together: $0=$ zero; $0.5=$ rare $(\sim 1-2$ cells per high power field (hpf)); $1=$ few $(\sim 3-4$ cells/hpf); $2=$ frequent $(\sim 5-10$ cells/hpf); $3=$ many $(\sim 11-20 / \mathrm{hpf}) ; 4=$ over $20 / \mathrm{hpf}$. For neutrophils (PMN), $5=$ sheets of PMN associated with alveolar wall destruction. The cells evaluated were: marginating $P M N$ in 
vasculature; PMN in alveolar spaces; mononuclear cells around conducting airways; and dead epithelial cells in conducting airways (mostly clusters).

Immunohistochemistry

IHC staining for the mature GRP peptide (a PNEC granule marker) and PGP 9.5 (the neural/NE cytoplasmic isoform of ubiquitin-Cterminal hydrolase-1) was carried out as described previously. ${ }^{14}$ In brief, formalin-fixed, paraffin-embedded lung sections were treated for $10 \mathrm{~min}$ with Triton X-100 $(0.3 \%$ in phosphatebuffered saline (PBS)), then normal serum blocking. Diluted primary antibodies were added to sections overnight at $4{ }^{\circ} \mathrm{C}$, followed by washing in PBS and incubation for $2 \mathrm{~h}$ at $4{ }^{\circ} \mathrm{C}$ with 1:200 dilution of biotinylated secondary antibodies (Vector, Burlingame, CA, USA). After blocking in $3 \% \mathrm{H}_{2} \mathrm{O}_{2}$ in methanol, Vector $A B C$ Elite was applied to slides for $30 \mathrm{~min}$. Slides were developed by using diaminobenzidine and $\mathrm{H}_{2} \mathrm{O}_{2}$, and then counterstained in $2 \%$ aqueous methyl green. All slides were blinded for semi-quantitative analysis. Results are expressed as mean numbers of cells positive for PGP 9.5 or GRP per $\mathrm{mm}^{2}$ lung tissue section. Statistical analysis was carried out using a onetailed Student's $t$ test.

\section{Quantitative real-time PCR}

Total RNA isolation and quantitative real-time PCR (qRT-PCR) were performed as previously described. ${ }^{63}$ Levels of mRNA for specific genes were normalized to the level of the housekeeping gene, $H P R T$, in the same samples and are expressed as "fold increase" over the relative gene expression measured in mock-infected lungs.

\section{Cytokine measurements}

Cytokine levels were measured by enzyme-linked immunosorbent assay by the Cytokine Core Laboratory in the University of Maryland School of Medicine's Center for Innovative Biomedical Resources.

\section{Statistics}

Statistical differences between two groups were determined using an unpaired, one-tailed Student's $t$ test with significance set at $p<0.05$. For comparisons between $\geq 3$ groups, analysis was done by one-way analysis of variance followed by a Tukey's multiple comparison post hoc test with significance determined at $p<0.05$. For survival studies, a log-rank (Mantel-Cox) test was used.

\section{ACKNOWLEDGEMENTS}

We thank Dr. Alan Cross for his careful review of and suggestions for this manuscript. Cytokine levels were measured by the Cytokine Core Laboratory in the University of Maryland School of Medicine's Center for Innovative Biomedical Resources (CIBR). This work was supported by the following grants: NIH Al125215 and Al104541 (SNV/ JCGB).

\section{AUTHOR CONTRIBUTIONS}

All authors contributed to the experimental design of this study. Specifically, K.A.S. and W.L. carried out all mouse in vivo and in vitro experiments, M.E.S. was responsible for histopathology and imaging, M.C.P. and J.C.G.B. carried out cotton rat assays and virus titers, and F.C. and S.N.V. oversaw the project. All co-authors participated in the writing of this manuscript.

\section{ADDITIONAL INFORMATION}

The online version of this article (https://doi.org/10.1038/s41385-018-0081-9) contains supplementary material, which is available to authorized users.

Competing interests: The authors declare no competing interests.

\section{REFERENCES}

1. Jensen, R. T., Battey, J. F., Spindel, E. R. \& Benya, R. V. International union of pharmacology. LXVIII. Mammalian bombesin receptors: nomenclature, distribution, pharmacology, signaling, and functions in normal and disease states. Pharmacol. Rev. 60, 1-41 (2008).

2. Ramos-Álvarez, I. et al. Insights into bombesin receptors and ligands: highlighting recent advances. Peptides 72, 128-144 (2015).

3. Jaeger, N., Czepielewski, R. S., Bagatini, M., Porto, B. N. \& Bonorino, C. Neuropeptide gastrin-releasing peptide induces $\mathrm{PI} 3 \mathrm{~K} /$ reactive oxygen speciesdependent migration in lung adenocarcinoma cells. Tumor Biol. 39, 1-11 (2017).

4. Cuttitta et al. Bombesin-like peptides can function as autocrine growth factors in human small-cell lung cancer. Nature 316, 823-826 (1985).

5. Moody et al. BW2258U89: a GRP receptor antagonist which inhibits small cell lung cancer growth. Life Sci. 56, 521-529 (1995).

6. Martínez, A. et al. Identification of vasoactive nonpeptide positive and negative modulators of adrenomedulin using a neutralizing antibody-based screening strategy. Endocrinology 145, 3858-3865 (2004).

7. Martínez, A., Zudaire, E., Julián, M., Moody, T. W. \& Cuttitta, F. Gastrin-releasing peptide (GRP) induces angiogenesis and the specific GRP blocker 77427 inhibits tumor growth in vitro and in vivo. Oncogene 24, 4106-4113 (2005).

8. Sunday, M. E., Yoder, B. A., Cuttitta, F., Haley, K. J. \& Emanuel, R. L. Bombesin-like peptide mediate lung injury in a baboon model of bronchopulmonary dysplasia. J. Clin. Invest. 102, 584-594 (1998).

9. Gosney, J. R., Sissons, M. C., Allibone, R. O. \& Blakey, A. F. Pulmonary endocrine cells in chronic bronchitis and emphysema. J. Pathol. 157, 127-133 (1989).

10. Meloni, F. et al. Bombesin enhances monocyte and macrophage activities: possible role in the modulation of local pulmonary defenses in chronic bronchitis. Respiration 63, 28-34 (1996).

11. Sunday, M. W. Oxygen, gastrin-releasing peptide, and pediatric lung disease: life in the balance. Front. Pediatr. 72, https://doi.org/10.3389/fped.2014.00072 (2014).

12. Moody, T. W., Pert, C. B., Gazdar, A. F., Carney, D. N. \& Minna, J. D. High levels of intracellular bombesin charactize human small-cell lung carcinoma. Science 214, 1246-1248 (1981).

13. Subramaniam, M. et al. Bombesin-like peptides modulate alveolarization and angiogenesis in bronchopulmonary dysplasia. Am. J. Respir. Crit. Care Med. 176, 902-912 (2007).

14. Zhou, S. et al. Radiation-induced lung injury is mitigated by blockade of gastrinreleasing peptide. Am. J. Pathol. 182, 1248-1254 (2013).

15. Nhu, Q. M. et al. Novel signaling interactions between proteinase-activated receptor 2 and Toll-like receptors in vitro and in vivo. Mucosal Immunol. 3, 29-39 (2010).

16. Shirey, K. A. et al. The TLR4 antagonist Eritoran protects mice from lethal influenza infection. Nature 497, 498-502 (2013).

17. Shirey, K. A. et al. Novel strategies for targeting innate immune responses to influenza. Mucosal Immunol. 9, 1173-1182 (2016).

18. Piao, W. et al. A decoy peptide that disrupts TIRAP recruitment to TLRs is protective in a murine model of influenza. Cell. Rep. 11, 1941-1952 (2015).

19. Perrin-Cocon, L. et al. TLR4 antagonist FP7 inhibits LPS-induced cytokine production and glycolytic reprogramming in dendritic cells, and protects mice from lethal influenza infection. Sci. Rep. 7, https://doi.org/10.1038/srep40791 (2017).

20. Li, A., Potts-Kant, E. N., Garantziotis, S., Foster, W. M. \& Hollingsworth, J. W. Hyaluronon signaling during ozone-induced injury requires TLR4, MyD88, and TIRAP. PLOS ONE 6, https://doi.org/10.1371/journal.pone.0027137 (2011).

21. Mathews, J. A. et al. Augmented responses to ozone in obese mice require IL-17A and gastrin-releasing peptide. Am. J. Respir. Cell. Mol. Biol. 58, 341-351 (2018).

22. Petronilho, F. et al. Gastrin-releasing peptide receptor antagonism induces protection from lethal sepsis: involvement of toll-like receptor 4 signaling. Mol. Med. 18, 1209-1219 (2012)

23. Abdulkhalek, S., Guo, M., Amith, S. R., Jayanth, P. \& Szewczuk, M. R. G-protein coupled receptor antagonists mediate Neu1 sialidase and matrix metalloproteinase- 9 cross-talk to induce transactivation of TOL-like receptors and cellular signaling. Cell Signal. 24, 2035-2042 (2012).

24. Blanco, J. C. et al. Receptor characterization and susceptibility of cotton rats to avian and 2009 pandemic influenza virus strains. J. Virol. 87, 2036-2045 (2013).

25. Polak, J. M. et al. Lung endocrine cell markers, peptides, and amines. Anat. Rec. 236, 169-171 (1993).

26. Czepieleski, R. S. et al. Gastrin-releasing peptide receptor (GRPR) mediates chemotaxis in neutrophils. Proc. Natl. Acad. Sci. USA 109, 547-552 (2012).

27. Cutz, E., Chan, W., Wong, V. \& Conen, P. E. Ultrastructure and fluorescence histochemistry of endocrine (APUD-type) cells in tracheal mucosa of human and various animal species. Cell Tissue Res. 158, 425-437 (1975).

28. McDonald, T. et al. A gastrin releasing peptide from the porcine nonantral gastric tissue. Gut 19, 767-774 (1978). 
29. Schnabel, E., Mains, R. E. \& Farquhar, M. G. Proteolytic processing of pro-ACTH/ endorphin begins in the Golgi complex of pituitary corticotropes and AtT-20 cells. Mol. Endocrinol. 3, 1223-1235 (1989).

30. Blalock, J. E., Harbour-McMenamin, D. \& Smith, E. M. Peptide hormones shared by the neuroendocrine and immunologic systems. J. Immunol. 135, 858s-861s (1985).

31. Kiess, W. \& Belohradsky, B. H. Endocrine regulation of the immune system. Klin. Wochenschr. 64, 1-7 (1986).

32. Zudaire, E. et al. Adrenomedullin is a cross-talk molecule that regulates tumor and mast cell function during human carcinogenesis. Am. J. Pathol. 168, 280-291 (2006).

33. Erbelding, E. J. et al. A universal influenza vaccine: the strategic plan for the national institute of allergy and infectious diseases. J. Infect. Dis. 218, 347-354 (2018).

34. Rajão, D. S. \& Pérez, D. R. Universal vaccines and vaccine platforms to protect against influenza viruses in humans and agriculture. Front. Microbiol. 9, https:// doi.org/10.3389/fmicb.2018.00123 (2018).

35. Gubareva, L. V. et al. Global update on the susceptibility of human influenza viruses to neuraminidase inhibitors, 2015-2016. Antivir. Res. 146, 12-20 (2017).

36. Centers for Disease Control and Prevention (CDC). Update: influenza activityUnited States, October 1, 2017-February 3, 2018. Morb. Mortal. Wkly. Rep. 67 169-179 (2018)

37. Centers for Disease Control and Prevention (CDC). Antiviral agents for the treatment and chemoprohylaxis of influenza. Morb. Mortal. Recomm. Rep. 60, 1-26 (2011).

38. Yang, H. et al. MD-2 is required for disulfide HMGB1-dependent TLR4 signaling. J. Exp. Med. 212, 5-14 (2015).

39. Kong, Y. et al. Functional diversity of notch family genes in fetal lung development. Am. J. Lung Cell. Mol. Physiol. 286, L1075-L1083 (2004).

40. Li, C. et al. Apc deficiency alters pulmonary epithelial cell fate and inhibits Nkx2.1 via triggering TGF-beta signaling. Dev. Biol. 378, 13-24 (2013).

41. Emanual, R. L. et al. Bombesin-like peptides and receptors in normal fetal baboon lung: roles in lung growth and maturation. Am. J. Physiol. 227, L1003-L1017 (1999).

42. Shapira, S. D. et al. A physical and regulatory map of host-influenza interactions reveals pathways in H1N1 infection. Cell 139, 1255-1267 (2009).

43. Aguayo, S. M. Determinants of susceptibility to cigarette smoke. Potential roles for neuroendocrine cells and neuropeptides in airway inflammation, airway wall remodeling, and chronic airflow obstruction. Am. J. Crit. Care Med. 149, 1692-1698 (1994).

44. Pardo, A. et al. Increase of lung neutrophils in hypersensitivity pneumonitis is associated with lung fibrosis. Am. J. Respir. Crit. Care Med. 161, 1698-1704 (2000).

45. Segura-Valdez, L. et al. Upregulation of gelatinases A and B, collagenases 1 and 2 , and increased parenchymal cell dealth in COPD. Chest 117, 684-694 (2000).

46. Corbel, M., Biochot, E. \& Lagente, V. Role of gelatinases MMP-2 and MMP-9 in tissue remodeling following acute lung injury. Braz. J. Med. Biol. Res. 33, 749-754 (2000).
47. Mullarkey, M. et al. Inhibition of endotoxin response by E5564, a novel Tolllike receptor 4-directed endotoxin antagonist. J. Pharmacol. Exp. Ther. 304 1093-1102 (2003).

48. Kim, H. M. et al. Crystal structure of the TLR4-MD-2 complex with bound endotoxin antagonist Eritoran. Cell 130, 906-917 (2007).

49. Meloni, F. et al. In vitro effect of bombesin-related peptides on the procoagulant activity of alveolar macropahges. Monaldi Arch. Chest Dis. 50, 187-190 (1995).

50. Rallabhandi, P. et al. Analysis of proteinase-activated receptor and TLR4 signal transduction: a novel paradigm for receptor cooperativity. J. Biol. Chem. 283, 24314-24325 (2008).

51. Lui, V. W. Y. et al. Mitogenic effects of gastrin-releasing peptide in head and neck squamous cancer cells are mediated by activation of the epidermal growth factor receptor. Oncogene 22, 6183-6193 (2003).

52. Liu, X. et al. Gastrin-releasing peptide activates Akt through the epidermal growth factor receptor pathway and abrogates the effect of gefitinib. Exp. Cell Res. 313 1361-1372 (2007).

53. Moody, T. W. et al. Bombesin receptor subtype-3 agonists stimulated the growth of lung cancer cells and increases EGF receptor tyrosine phosphorylation. Peptides 32, 1677-1684 (2011).

54. Basu, S. et al. Helicobacter pylori HP0175 transactivates epidermal growth factor receptor through TLR4 in gastric epithelial cells. J. Biol. Chem. 283, 32369-32376 (2008).

55. Thuringer, D. et al. Transactivation of the epidermal growth factor receptor by heat shock protein 90 via toll-like receptor 4 contributes to the migration of glioblastoma cells. J. Biol. Chem. 286, 3418-3428 (2011).

56. McElory, S. J. et al. Transactivaton of EGFR by LPS induces COX-2 expression in enterocytes. PLoS ONE 7, https://doi.org/10.1371/journal.pone.0038373 (2012).

57. Chattopadhyay, S. et al. EGRF kinase activity is required for TLR4 signaling and the septic shock responses. EMBO Rep. 16, 1535-1547 (2015).

58. De, S. et al. Erlotinib protects against LPS-induced endotoxicity because TLR4 need EGFR to signal. Proc. Natl. Acad. Sci. USA 112, 9680-9685 (2015).

59. Yamaguchi, R. et al. Neutrophil elastase enhances IL-20 production by lipopolysaccharide-stimulated macrophages via transactivation of the PAR-2/ EGFR/TLR4 signaling pathway. Blood Cells Mol. Dis. 59, 1-7 (2016).

60. Teijaro, J. R. et al. Costimulation modulation uncouples protection from immunopathology in memory $\mathrm{T}$ cell response to influenza virus. J. Immunol. 182 6834-6843 (2009).

61. Ottolini, M. G. et al. The cotton rat provides a useful small-animal model for the study of influenza virus pathogenesis. J. Gen. Virol. 86, 2823-2830 (2005).

62. Patel, M. C., Shirey, K. A., Boukhvalova, M. S., Vogel, S. N. \& Blanco, J. C. G. Serum high-mobility-group box 1 as a biomarker and a therapeutic target during respiratory virus infections. mBio 9, https://doi.org/10.1128/mBio.00246-18 (2018).

63. Shirey, K. A., Cole, L. E., Keegan, A. D. \& Vogel, S. N. Francisella tularensis live vaccine strain induces macrophage alternative activation as a survival mechanism. J. Immunol. 181, 4159-4167 (2008). 